\title{
To Explore the Mechanism of Prunella Vulgaris on Lymphoma Based on Network Pharmacology and Molecular Docking
}

\author{
Xiaohong $\mathrm{Wu}^{1,2}$, Huiling Song ${ }^{2,3}$, Aixia Sui ${ }^{2, *}$, Xinyu Zhao ${ }^{1,2}$, Hongtao Zhang ${ }^{1}$ \\ ${ }^{1}$ College of Postgraduate, Hebei North University, Zhangjiakou, China \\ ${ }^{2}$ Hebei General Hospital, Shijiazhuang, China \\ ${ }^{3}$ College of Postgraduate, North China University of Science and Technology, Tangshan, China
}

\section{Email address:}

xiaohongtougao@163.com (Xiaohong Wu),shl1031086205@163.com (Huiling Song), suiaxhebei@126.com (Aixia Sui), zxyzb2020@163.com (Xinyu Zhao), hongtaozhangmd@163.com (Hongtao Zhang)

${ }^{*}$ Corresponding author

\section{To cite this article:}

Xiaohong Wu, Huiling Song, Aixia Sui, Xinyu Zhao, Hongtao Zhang. Research paper To Explore the Mechanism of Prunella Vulgaris on Lymphoma Based on Network Pharmacology and Molecular Docking. Cancer Research Journal. Vol. 9, No. 3, 2021 , pp. $154-161$. doi: $10.11648 /$ j.crj.20210903.14

Received: June 22, 2021; Accepted: July 2, 2021; Published: July 13, 2021

\begin{abstract}
Background. Although the traditional Chinese medicine Prunella vulgaris may be effective in treating lymphoma, its mechanism of action remains unclear. The purpose of this paper is to explore the mechanism of Prunella vulgaris against lymphoma by combining network pharmacology and molecular docking. Methods. The potential active ingredients of Prunella vulgaris were queried in the TCMSP database. Lymphoma-related genes were searched in the DisgeNet database. After removing the duplicates, the remaining active targets were compared with lymphoma genes to obtain the key target and analyzed using a Venn map. Building a network of protein interactions through String platforms. The GO and KEGG databases were used for enrichment analysis of the key targets with the help of the DAVID analysis platform. Results. A total of 39 potentially active components and 125 targets were identified from Prunella vulgaris, 7592 lymphoma-related target genes, and 101 key target genes for the intersection of Prunella vulgaris and lymphoma. GO entries were related to biological processes including enzyme binding, protein binding, positive regulation of transcription, regulation of cell proliferation, and negative regulation of cell death. KEGG analysis identified the signalling pathways of HIF-1, estrogen, NOD-like receptors, PI3K-Akt, and TNF. The binding between the selected compounds and the target molecules was modelled by molecular docking. Conclusion. Through network pharmacology, it is predicted that Prunella vulgaris may regulate multiple signalling pathways through numerous targets, and thereby affect the functions of multiple cells and playing a role in the treatment of lymphoma.
\end{abstract}

Keywords: Prunella Vulgaris, Lymphoma, Network Pharmacology, Molecular Docking

\section{Introduction}

Lymphoma is a common malignancy of the immune system originating from lymph nodes or extranodal lymphoid tissue. Most of its occurrence is related to the malignancy of certain immune cells produced by the proliferation and differentiation of lymphocytes during the immune response. It is generally believed that infection and immune factors play essential roles and physical, chemical, and genetic factors. Lymphoma is a relatively rare but highly curable malignant tumor. Chemotherapy, radiotherapy, targeted therapy, and biological therapy are the current main therapies. However, the effectiveness of traditional Chinese medicine (TCM) treatment for lymphoma has been recently reported [1, 2], but the mechanism of action has not been clearly explained. This might be because there are many components in TCM and their biochemical mechanisms might be complex. Therefore, it is prudent to analyze the molecular mechanisms of TCM using network pharmacology research.

Prunella vulgaris is a species of perennial herb in the Lamiaceae family. Among the genus Prunella, Prunella 
vulgaris is the most studied and has been used for thousands of years in antipyretic and detoxifying Chinese medicine. Prunella vulgaris has many active components [3] and a wide range of pharmacological effects $[4,5]$, including antibacterial and anti-inflammatory activity [6], lowering blood pressure, and liver protection. Prunella vulgaris is also a TCM with anti-cancer $[7,8]$ and strong antioxidant effects, low toxicity, a high safety factor, and multiple targets [9]. Prunella vulgaris has been shown to have therapeutic properties in a variety of tumours.

With the emergence of the concept of network pharmacology, a new method has been opened up for the study of using TCM to treat diseases [10]. TCMs have broad prospects for development in the treatment of complex diseases [11]. However, TCMs have complex mechanisms of action, which are poorly understood at the molecular level due to the lack of modern research using bioinformatics, pharmacology, and molecular biology, and TCM treatment remains problematic [12]. Network pharmacology is a discipline that uses computer analysis and database to carry out multi-target molecular analysis. It can reveal complex disease relationship through multi-signal pathway and multi-pathway regulation, and at the same time improve drug treatment effect and reduce side effects. It is systematic and can analyze the effects of drug interventions on disease to reveal the complex pharmacodynamic reactions in the human body [13]. This allows more accurate prediction and analysis of the mechanism of action of TCMs. Molecular docking studies between receptors and ligands can predict their binding affinities and test their interactions allowing for rational drug design [14].

\section{Materials and Methods}

\subsection{Identification and Target Prediction of Prunella Vulgaris Active Components}

Using the TCMSP database, the keyword "Prunella vulgaris" was used to find the active components of Prunella vulgaris. After $\mathrm{OB} \geq 30 \%$ and $\mathrm{DL} \geq 0.18$ were set as the limits, the effective active ingredients of Prunella vulgaris were identified, and the potential targets were obtained from the UniProt database.

\subsection{Lymphoma-Related Targets and Venn Map Analysis}

Lymphoma-related genes were retrieved using the GeneCards database with "Lymphoma" as the keyword. The potential targets of Prunella vulgaris and lymphoma were compared through the Venn database, and the intersecting genes (key target genes) were obtained and plotted using Venn diagrams.

\subsection{Construction of Protein Interaction Networks}

The key target genes were imported into the STRING database, a protein interaction (PPI) network was constructed, and the PPI pictures were saved.

\subsection{GO and KEGG Analysis}

Key target genes were imported into the DAVID database for GO and KEGG pathway analyses to study the main metabolic pathways and biological processes of Prunella vulgaris components in the treatment of lymphoma targets, and to analyze the biological significance of the intersection genes.

\section{5. "Component-target" Diagram Construction}

The active ingredients and targets were imported into Cytoscape 3.6.0 software to build a visual association between components and targets.

\subsection{Molecular Docking}

The three-dimensional structure of the compound was found from the PubChem database, and the key receptor proteins were found from the Protein database. Proteins were pretreated to remove water molecules using Autotools. AutoGrid was used to calculate the energy grid points. AutoDock was used to dock small molecules with the proteins, and the binding energy score was calculated for each docking. Visualization was performed using DS and PyMol.

\section{Results}

\subsection{Target Prediction of Chemical Constituents in Prunella Vulgaris}

Using the TCMSP database, 39 potential active components and 489 targets of Prunella vulgaris were screened using "Prunella vulgaris" as the key word. The UniProt database was used to obtain 125 corrected targets after integration and reduplication.

\subsection{Key Targeting of Lymphoma and Drawing Venn map}

A total of 7592 lymphoma-related target genes were collected through the GeneCards platform, and 101 key target genes were obtained after intersection with Prunella vulgaris target. Critical targets are mainly Bcl-2, IL-6, RB1, IL-2, CDK4, CDK6, ABCB1, EGFR, and VEGFA. Draw the Venn map (Figure 1).

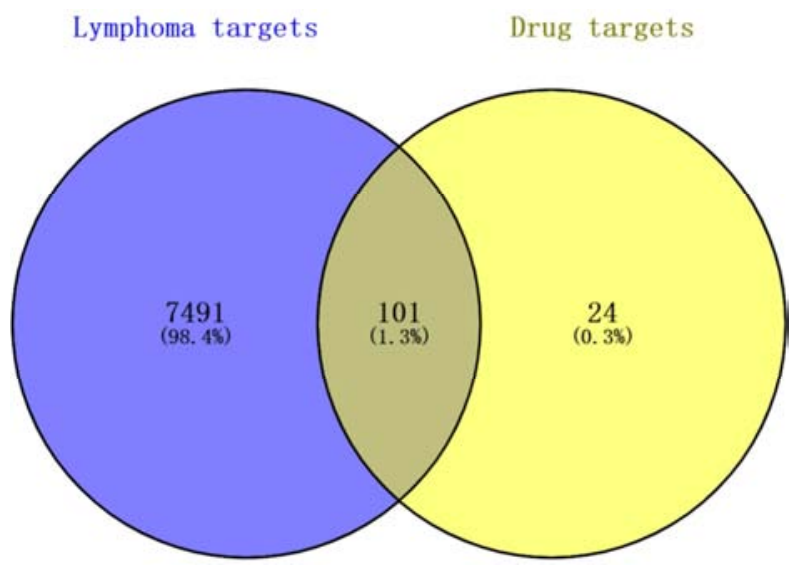

Figure 1. Venn charts of constituent and disease targets. The blue circles represent the lymphoma genes and the yellow ones represent the prunella vulgaris genes. The intersection of the two is the key target. 


\subsection{Protein Interaction Network Analysis}

The key targets were imported into the STRING database. The study species were selected as human, the interaction score was set to greater than 0.9 , and the protein-gene interaction relationship was obtained (Figure 2). The TSV format was chosen to export the results, and the data was imported into the Cytoscape network topology analysis software. The top 7 targets were INS, IL-6, VEGFA, JUN, EGF, EGFR, and ESR1. These targets occupy a key position in the PPI network diagram, suggesting that these seven targets are the key points of Prunella vulgaris in the treatment of lymphoma.

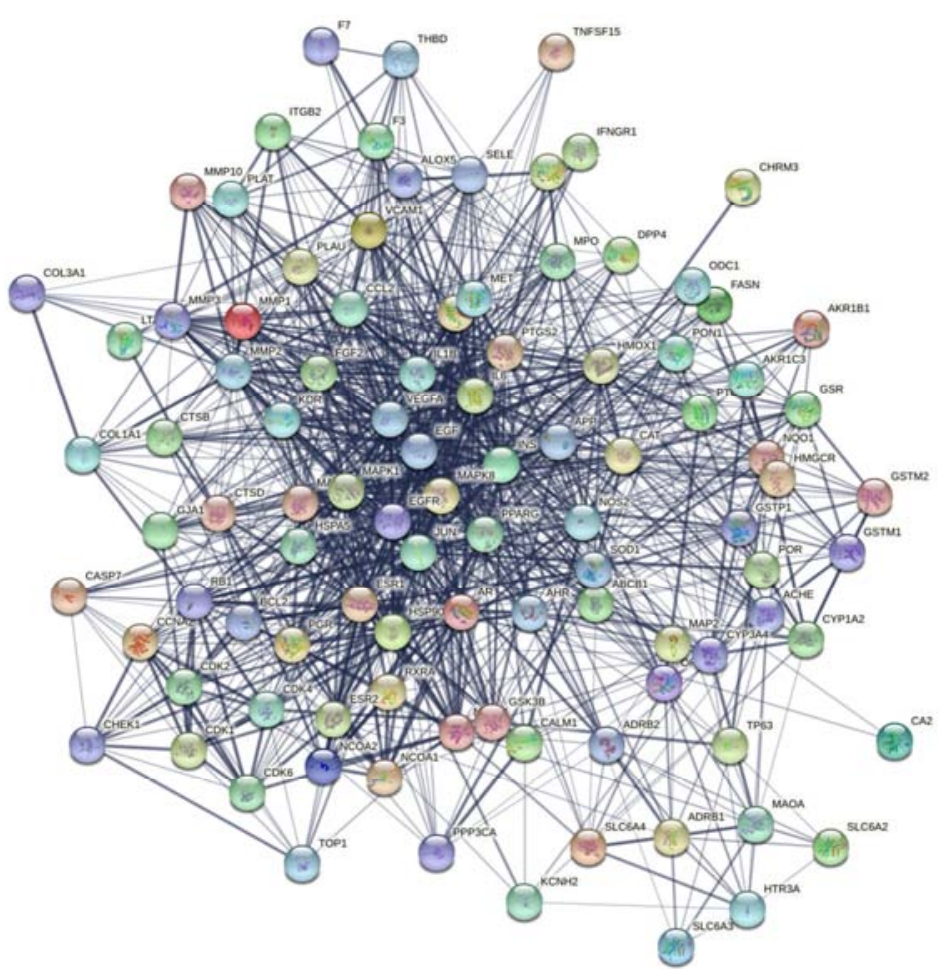

Figure 2. Key target gene network interaction.

\subsection{GO Pathway Analysis}

The GO biological processes of the key targets were analyzed (Figure 3), and a total of $465 \mathrm{GO}$ entries were obtained. The top $20 \mathrm{GO}$ annotations were screened according to the Count values (Table 1), 13 of which were related to biological processes (BP), 3 to molecular functions (MF), and 4 to cellular components (CC). The GO entries mainly were related to biological processes that included protein binding, positive regulation of transcription, positive regulation of smooth muscle cell proliferation, regulation of cell proliferation, and negative regulation of cell death.

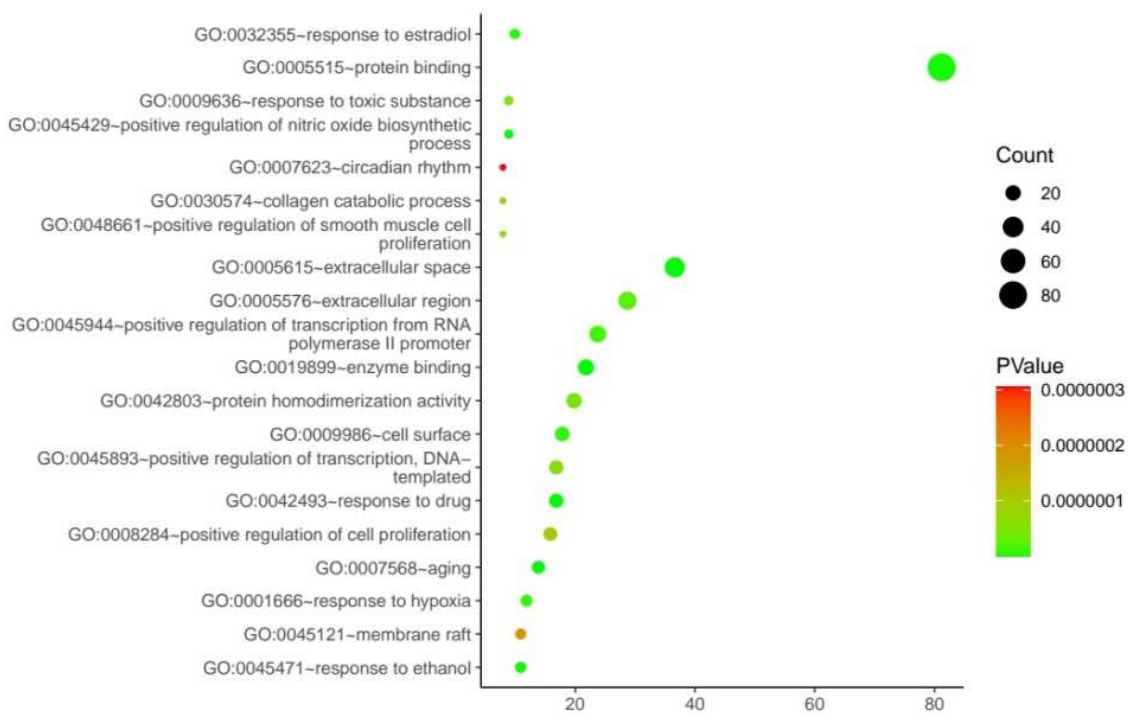

Figure 3. The vertical axis is the main biological process, and the horizontal-axis is the richness of the various factors. 
Table 1. Go data graph.

\begin{tabular}{|c|c|c|c|c|}
\hline NO & Category & Term & Count & PValue \\
\hline 1 & GOTERM_MF_DIRECT & GO: 0019899 enzyme binding & 22 & $3.88 \mathrm{E}-16$ \\
\hline 2 & GOTERM_MF_DIRECT & GO: $0005515 \sim$ protein binding & 82 & $1.45 \mathrm{E}-09$ \\
\hline 3 & GOTERM_MF_DIRECT & GO: $0042803 \sim$ protein homodimerization activity & 20 & 4.69E-08 \\
\hline 4 & GOTERM_CC_DIRECT & GO: 0005615 extracellular space & 37 & $1.98 \mathrm{E}-16$ \\
\hline 5 & GOTERM_CC_DIRECT & GO: $0009986 \sim$ cell surface & 18 & 5.88E-09 \\
\hline 6 & GOTERM_CC_DIRECT & GO: 0005576 extracellular region & 29 & $2.31 \mathrm{E}-08$ \\
\hline 7 & GOTERM_CC_DIRECT & GO: $0045121 \sim$ membrane raft & 11 & $1.96 \mathrm{E}-07$ \\
\hline 8 & GOTERM_BP_DIRECT & GO: $0007568 \sim$ aging & 14 & $1.68 \mathrm{E}-11$ \\
\hline 9 & GOTERM_BP_DIRECT & GO: $0042493 \sim$ response to drug & 17 & 3.08E-11 \\
\hline 10 & GOTERM_BP_DIRECT & GO: 0045429 positive regulation of nitric oxide biosynthetic process & 9 & $1.45 \mathrm{E}-10$ \\
\hline 11 & GOTERM_BP_DIRECT & GO: $0045471 \sim$ response to ethanol & 11 & $6.41 \mathrm{E}-10$ \\
\hline 12 & GOTERM_BP_DIRECT & GO: $0032355 \sim$ response to estradiol & 10 & 3.42E-09 \\
\hline 13 & GOTERM_BP_DIRECT & GO: $0001666 \sim$ response to hypoxia & 12 & $6.08 \mathrm{E}-09$ \\
\hline 14 & GOTERM_BP_DIRECT & GO: $0045944 \sim$ positive regulation of transcription from RNA polymerase II promoter & 24 & $1.10 \mathrm{E}-08$ \\
\hline 15 & GOTERM_BP_DIRECT & GO: $0009636 \sim$ response to toxic substance & 9 & 3.93E-08 \\
\hline 16 & GOTERM_BP_DIRECT & GO: 0045893 positive regulation of transcription, DNA-templated & 17 & $6.08 \mathrm{E}-08$ \\
\hline 17 & GOTERM_BP_DIRECT & GO: 0048661 positive regulation of smooth muscle cell proliferation & 8 & $6.41 \mathrm{E}-08$ \\
\hline 18 & GOTERM_BP_DIRECT & GO: $0030574 \sim$ collagen catabolic process & 8 & $1.01 \mathrm{E}-07$ \\
\hline 19 & GOTERM_BP_DIRECT & GO: $0008284 \sim$ positive regulation of cell proliferation & 16 & $1.04 \mathrm{E}-07$ \\
\hline 20 & GOTERM_BP_DIRECT & GO: $0007623 \sim$ circadian rhythm & 8 & $3.06 \mathrm{E}-07$ \\
\hline
\end{tabular}

\subsection{KEGG Pathway Analysis}

After using David database to analyze the key targets, it can be seen that the targets are mainly concentrated in the signalling pathways of HIF-1, estrogen, NOD-like receptors, PI3K-Akt, and TNF (Figure 4). The top 20 KEGG annotations were screened based on the $\mathrm{P}$ values (Table 2). These pathways are involved in the inflammatory, hormonal, and immune factor signaling responses in lymphoma.

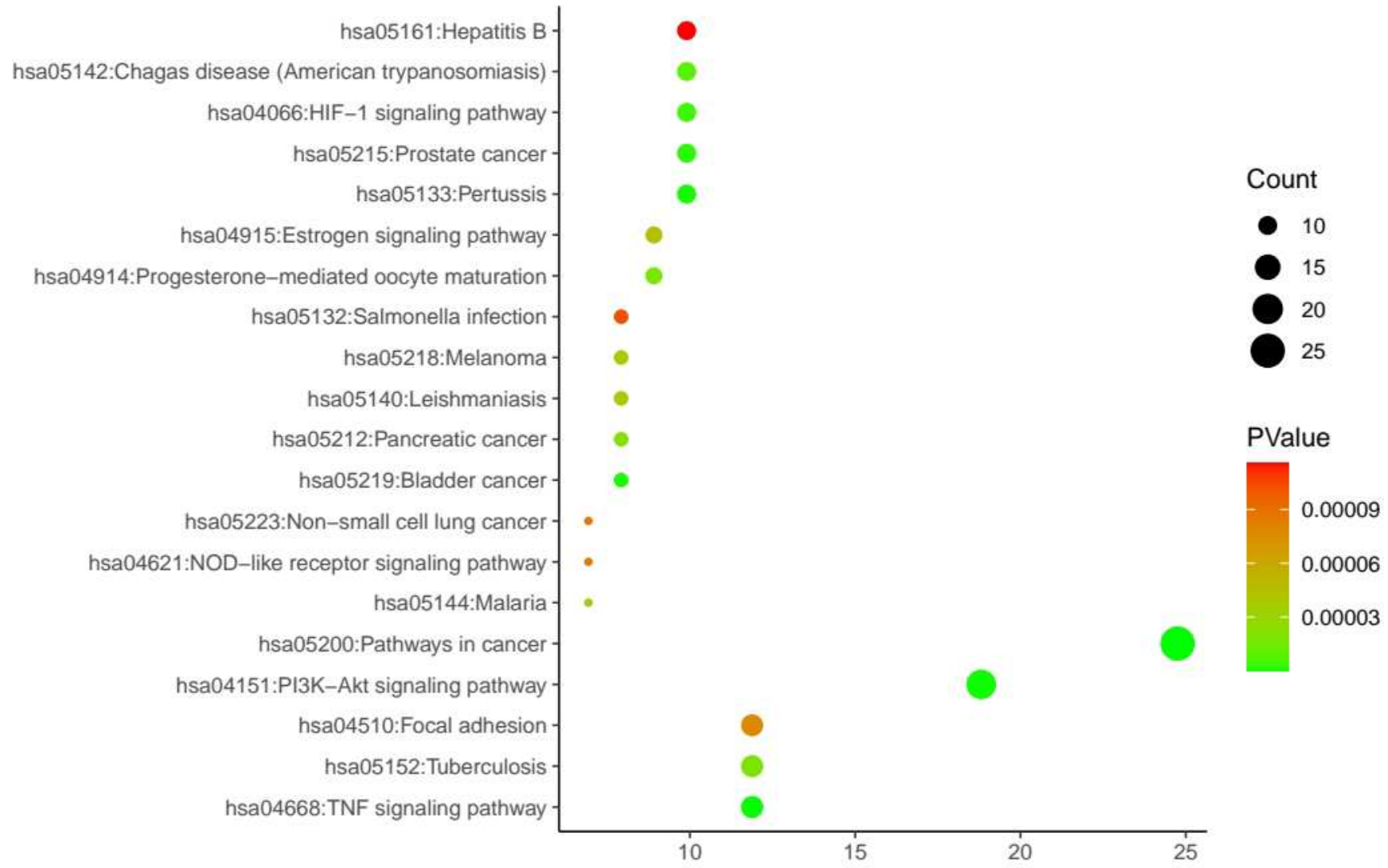

Figure 4. The vertical-axis is the main signalling pathway, and the horizontal-axis is the abundance of factors. 
Table 2. KEGG data graph.

\begin{tabular}{llll}
\hline NO & Term & Count & PValue \\
\hline 1 & hsa05200: Pathways in cancer & 25 & $1.26 \mathrm{E}-10$ \\
2 & hsa04151: PI3K-Akt signaling pathway & 19 & $4.26 \mathrm{E}-07$ \\
3 & hsa04668: TNF signaling pathway & 12 & $1.29 \mathrm{E}-07$ \\
4 & hsa05152: Tuberculosis & 12 & $1.90 \mathrm{E}-05$ \\
5 & hsa04510: Focal adhesion & 12 & $7.71 \mathrm{E}-05$ \\
6 & hsa05133: Pertussis & 10 & $5.10 \mathrm{E}-07$ \\
7 & hsa05215: Prostate cancer & 10 & $2.02 \mathrm{E}-06$ \\
8 & hsa04066: HIF-1 signaling pathway & 10 & $4.20 \mathrm{E}-06$ \\
9 & hsa05142: Chagas disease (American trypanosomiasis) & $8.16 \mathrm{E}-06$ \\
10 & hsa05161: Hepatitis B & 10 & $1.16 \mathrm{E}-04$ \\
11 & hsa04914: Progesterone-mediated oocyte maturation & 10 & $1.71 \mathrm{E}-05$ \\
12 & hsa04915: Estrogen signaling pathway & 9 & $4.40 \mathrm{E}-05$ \\
13 & hsa05219: Bladder cancer & 9 & $8.76 \mathrm{E}-07$ \\
14 & hsa05212: Pancreatic cancer & 8 & $2.10 \mathrm{E}-05$ \\
15 & hsa05140: Leishmaniasis & 8 & $3.76 \mathrm{E}-05$ \\
16 & hsa05218: Melanoma & 8 & $3.76 \mathrm{E}-05$ \\
17 & hsa05132: Salmonella infection & 8 & $1.03 \mathrm{E}-04$ \\
18 & hsa05144: Malaria & 8 & $7.02 \mathrm{E}-05$ \\
19 & hsa04621: NOD-like receptor signaling pathway & 7 & $8.66 \mathrm{E}-05$ \\
20 & hsa05223: Non-small cell lung cancer & 7 & $8.66 \mathrm{E}-05$ \\
\hline
\end{tabular}

\subsection{Analysis of "Component-Target" Network Construction}

Target genes corresponding to 39 components and 489 components were imported into Cytoscape software for network construction and visualization, and the images were saved (Figure 5). The top 7 ingredients by degree were quercetin, kaempferol, beta-sitosterol, stigmasterol, luteolin, situogluside, and ursolic acid.

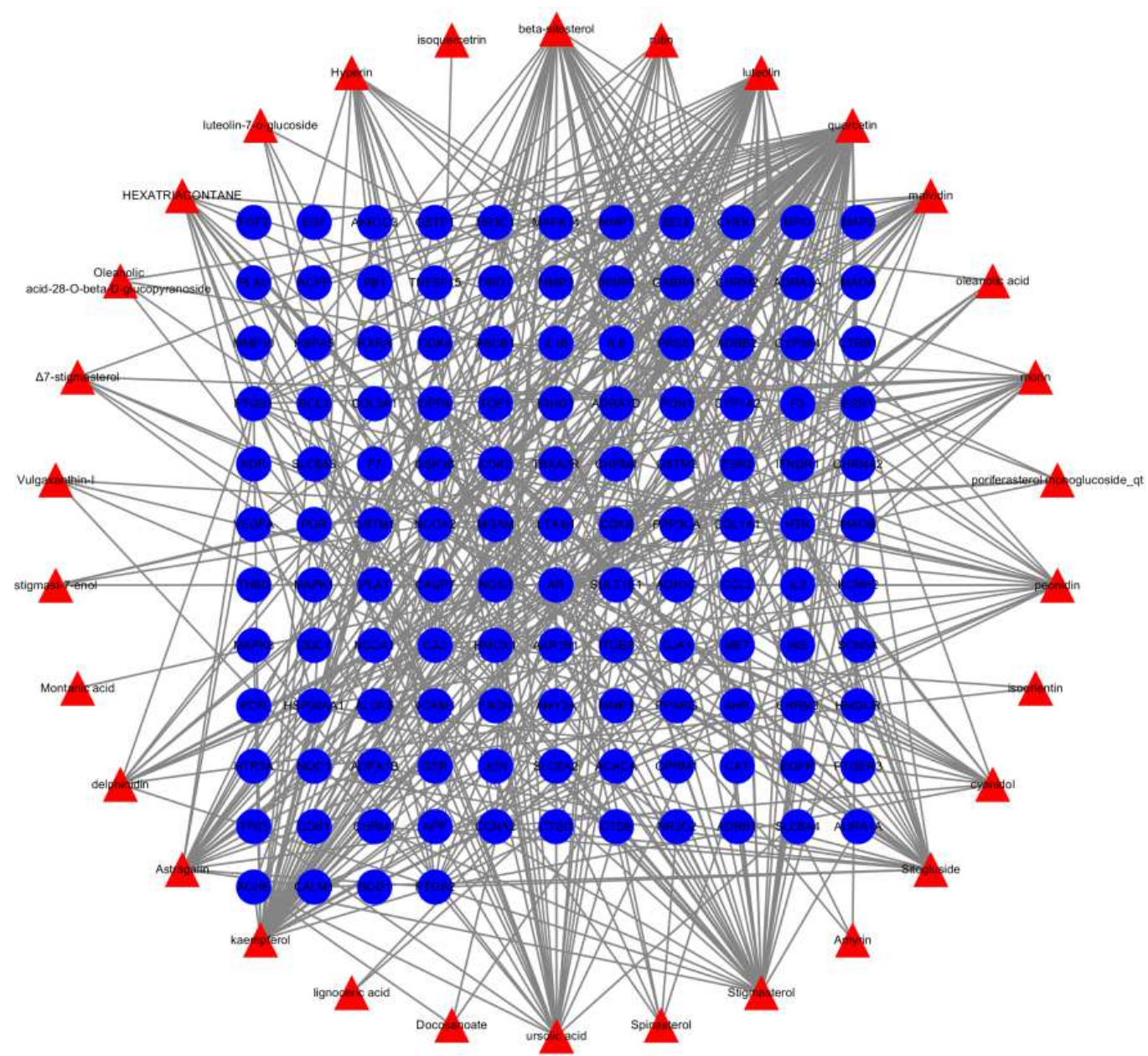

Figure 5. "component-target" network diagram. The blue circle represents the action targets and the red triangle represents the active component of Prunella vulgaris. 


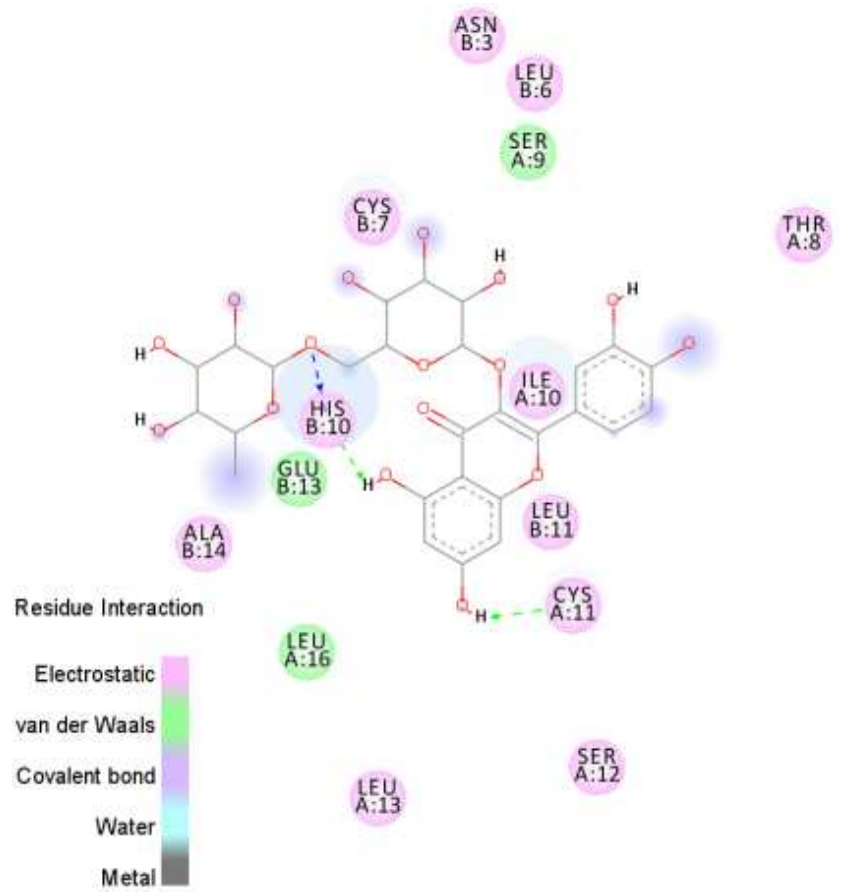

Figure 6. 2D diagram.

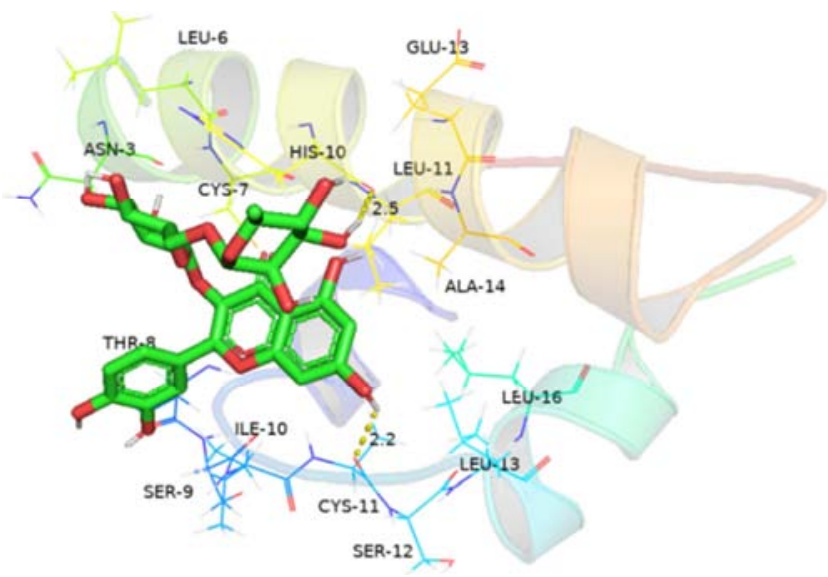

Figure 7. 3D diagram.

(The 3D and 2D images show van der Waals forces in light green, hydrogen bonds in green, undesirable ligands in red, cation and cation of Pi in orange, and alkylation of Pi in light purple.)

\subsection{Molecular Docking}

As shown in Figures 6 and 7, the ligand HisB: 10 formed a hydrogen bond and an ether bond with the hydroxyl group of rutin, and the ligand CysA: 11 formed a hydrogen bond with the hydroxyl group of rutin. The ligands HisB: 10 and CysA: 11 formed hydrogen bonds with rutin at a distance of 2.2 , and 2.5 , respectively. The binding energy $(-6.6 \mathrm{kcal} / \mathrm{mol})$, indicated that rutin and INS (PDB ID: 1UZ9) produced strong hydrogen bonds. This suggests that rutin, a key component of Prunella vulgaris, may play a therapeutic role through the target gene, INS.

\section{Discussion}

We used network pharmacology to analyze the components, targets, and related signalling pathways of Prunella vulgaris, and to explore the correlation between Prunella vulgaris and lymphoma. First, 39 main active ingredients were mined from the TCMSP database. Cytoscape software was used to determine that the main anti-lymphoma components of Prunella vulgaris were quercetin, kaempferol, and beta-sitosterol. Through the GeneCard database, we identified lymphoma-related genes, and compared with the active ingredients of Prunella vulgaris on the website, obtaining 101 duplicates of the genes. It can be seen from network topology analysis, the top targets were INS, IL-6, VEGFA, JUN, EGF, EGFR, and ESR1. Enrichment analysis of key targets suggests that Prunella vulgaris antagonism to lymphoma acts through key signalling pathway including the HIF-1, estrogen, and NOD-like receptor pathways. Therefore, Prunella vulgaris antagonism mainly acts on multiple targets, multiple pathways, and effects related signalling pathways to exert its pharmacological effects.

Lymphoma is a relatively complex malignant tumour that originates in the hematopoietic system. There is currently no clear evidence for the aetiology of lymphoma, but it is believed that the interaction of host immune function, infectious factors, and environmental factors contribute to the development of lymphoma. According to Globocan 2018 statistics [15], lymphomas accounted for $3.2 \%$ of the 18.1 million new cancer cases in 2018 and $2.9 \%$ of the 9.6 million cancer deaths in 2018. The number of new cases and deaths associated with lymphoma increased in 2018 compared to Globocan 2012 [16].

Estrogen plays a role in target blocking mainly through estrogen receptor $\alpha$ and estrogen receptor $\beta[17,18]$. Studies have demonstrated that ER $\beta$ agonists can be used to slow lymphoma growth and inhibit the spread of lymphoma angiogenesis [19]. Thus, the estrogen signalling pathway plays an important role in the fight against lymphoma. HIF (hypoxia-inducible factor) is composed of two subunits, HIF- $1 \alpha$ and HIF-1 $\beta$. A literature review shows that HIF- $1 \alpha$ mediated gene transcriptional regulation is involved in cell hypoxia [20]. Some studies have shown that HIF-1 $\alpha$ plays a vital role in the anti-lymphoma pathway of Prunella vulgaris, but further studies are needed to confirm its mechanism. The PI3K-Akt pathway is the main signalling pathway, and it is frequently activated in cancer. PI3K-mediated signal transduction is significant in malignant transformation. Akt can regulate the activity of various tumour markers to promote tumorigenesis and metastasis. Overactivation of AKT1 and glycolytic enzyme LDH-A and inactivation of tumour suppressor gene p53 lead to tumour cell proliferation and cell survival. Quercetin can prevent lymphoma by downregulating the PI3K - AKT1 - p53 pathway and glycolytic metabolism [21]. Moreover, quercetin does not 
exert any cytotoxicity on normal B cells, which may be why quercetin is better in the treatment of lymphoma [22]. TNF is a cytokine with a wide range of biological effects, divided into TNF- $\alpha$ and TNF- $\beta$, both of which are indispensable in the differentiation and maturation of B cells [23]. TNF- $\alpha$ is thought to play a protective role in the development of lymphoma [24], which may be an important reason for Prunella vulgaris in lymphoma through the TNF signaling pathway. NOD-like receptors have been shown to play an essential role in immune and infectious pathways [25].

Relevant literature has reported that the main component of natural drugs is ursolic acid, which has activity against blood tumour cells. In vitro studies have found that ursolic acid can inhibit the proliferation of lymphoma cells, induce cell differentiation, and promote apoptosis [26]. Luteolin is a yellow ketone, which exists in many foods, has powerful anticancer ability, has been widely used in a variety of human malignant tumours [27], can trigger apoptosis, inhibits the growth of cells, stimulates the stagnation of the cell cycle, and prevents cell migration. Studies have proved that luteolin is induced by caspase activation, causing cell death due to cell toxicity [28]. Kaempferol has been shown to exert anticancer activity through multiple pathways, such as inhibiting angiogenesis and expression of vascular endothelial growth factor (VEGF), inducing apoptosis, and regulating HIF-1 $\alpha$ [29].

IL-6 can promote the formation of tumour blood vessels, which is conducive to the growth and metastasis of cancer cells, and experiments have shown that IL-6 expression affects the progression of mouse thymus lymphoma [30]. VEGFA plays a key regulatory role in the growth and development of solid tumours and hematologic malignancies, mainly through binding to the receptors VEGFR1 and VEGFR2, in which VEGFR2 plays a major role in downstream angiogenesis [31]. In addition, VEGFA is closely associated with the prognosis of non-Hodgkin's lymphoma [32]. A review of relevant literature found that the main cause of the invasive phenotype of diffuse large B-cell lymphoma was the activation of JUN signalling [33]. Tumour cells and stromal cells play key roles in the development of malignant hematologic diseases. After EGF is exposed to lymphoma cells, stromal cells induce the expression of EGR-1, which may offer a new target for therapeutic intervention for lymphatic tumours [34]. These molecular targets are highly correlated with the occurrence and development of lymphoma.

\section{Conclusions}

We analyzed the anti-lymphoma mechanism of Prunella vulgaris at the molecular level through network pharmacology. The main action relationships of Prunella vulgaris against lymphoma were explored and suggested target molecules for further exploration of the in vivo pharmacological mechanism of Prunella vulgaris in the treatment of lymphoma. However, further cell or animal studies are needed to validate the visual associations between targets and pathways to further consolidate the role of Prunella vulgaris in the treatment of lymphoma.

\section{Data Availability}

The data of this study are attached on the manuscript.

\section{Conflicts of Interest}

All the authors do not have any possible conflicts of interest.

\section{Authors' Contributions}

Xiaohong $\mathrm{Wu}$, Huiling Song and Xinyu Zhao were responsible for collecting and processing the data. Xiaohong $\mathrm{Wu}$ was in charge of writing and revising the article, while Aixia Sui and Hongtao Zhang were in charge of reviewing the format. All authors discussed, edited, and approved the final manuscript.

\section{Acknowledgements}

We thank the Clinical Research Center of Hebei General Hospital, Guipei Base, for providing the experimental platform for this study.

\section{References}

[1] Y. Hijikata, J. Kaneko, L. Xi, et al. Traditional Chinese medicines improve the course of refractory leukemic lymphoblastic lymphoma and acute lymphocytic leukemia: two case reports, Am. J. Chin. Med. 23 (2) (1995) 195-211.

[2] H. Wang, F. Wang, S. Wu, Traditional herbal medicine-derived sulforaphene promotes mitophagic cell death in lymphoma cells through CRM1-mediated p62/SQSTM1 accumulation and AMPK activation, Chem. Biol. Interact. 281 (2018) 11-23.

[3] X. Gu, Y. Li, J. Mu, et al. Chemical constituents of Prunella vulgaris, J. Environ. Sci. (China), 25 (2013) 161-163.

[4] E. H. Han, J. H. Choi, Y. P. Hwang, et al. Immunostimulatory activity of aqueous extract isolated from Prunella vulgaris. Food \& Chemical Toxicology, 47 (1) (2009) 62-69.

[5] G. Meng, M. Wang, K. Zhang, et al. Research Progress on the Chemistry and Pharmacology of Prunella vulgaris Species, Oalib. Journal. 2014 (3) (2014) 1-19.

[6] S. Y. Ryu, M. H. Oak, S. K. Yoon, et al. Anti-allergic and anti-inflammatory triterpenes from the herb of Prunella vulgaris, Planta. Med, 66 (4) (2000) 358-360.

[7] Y. Fang, L. Zhang, J. Feng, et al. Spica Prunellae extract suppresses the growth of human colon carcinoma cells by targeting multiple oncogenes via activating miR-34a, Oncol. Rep. 38 (3) (2017) 1895-1901.

[8] D. T. Yin, M. Lei, J. Xu, et al. The Chinese herb Prunella vulgaris promotes apoptosis in human well-differentiated thyroid carcinoma cells via the B-cell lymphoma-2/Bcl-2-associated $\mathrm{X}$ protein/caspase-3 signaling pathway, Oncol. Lett. 14 (2) (2017) 1309-1314. 
[9] Y. J. HWANG, E. J. LEEi, H. R. KIM, et al. In vitro antioxidant and anticancer effects of solvent fractions from Prunella vulgaris var. lilacina, BMC. Complement. Altern. Med, 13 (1) (2013) 310.

[10] A. L. Hopkins, Network pharmacology, Nature. Biotechnology, 25 (10) (2007) 1110-1111.

[11] Z. H. Liu, X. B. Sun, [Network pharmacology: new opportunity for the modernization of traditional Chinese medicine], Yao. Xue. Xue. Bao, 47 (6) (2012) 696-703.

[12] W. Y. Lee, C. Y. Lee, Y. S. Kim, et al. The Methodological Trends of Traditional Herbal Medicine Employing Network Pharmacology, Biomolecules, 9 (8) (2019) 362.

[13] A. L. Hopkins, Network pharmacology: the next paradigm in drug discovery, Nat. Chem. Biol, 4 (11) (2008) 682-690.

[14] A. Bortolato, M. Fanton, S. J. Mason, et al. Molecular docking methodologies, Methods. Mol. Biol, 34 (2013) 339-360.

[15] F. Bray, J. Ferlay, I. Soerjomataram, et al. Global cancer statistics 2018: GLOBOCAN estimates of incidence and mortality worldwide for 36 cancers in 185 countries, CA. Cancer. J. Clin, 68 (6) (2018) 394-424.

[16] L. A. Torre, F. Bray, R. L. Siegel, et al. Global cancer statistics, 2012, CA. Cancer. J. Clin, 65 (2) (2015) 87-108.

[17] G. G. Kuiper, E. Enmark, M. Pelto-Huikko, et al. Cloning of a novel receptor expressed in rat prostate and ovary, Proc. Natl. Acad. Sci. U. S. A, 93 (12) (1996) 5925-5930.

[18] S. Nilsson, S. Mäkelä, E. Treuter, et al. Mechanisms of estrogen action, Physiol. Rev, 81 (4) (2001) 1535-1565.

[19] K. Yakimchuk, S. M. Hasni, J. Guan, et al. Inhibition of lymphoma vascularization and dissemination by estrogen receptor $\beta$ agonists, Blood, 123 (13) (2014) 2054-2061.

[20] V. Nizet, R. S. Johnson, Interdependence of hypoxic and innate immune responses, Nat. Rev. Immunol, 9 (9) (2009) 609-617.

[21] Akhilendra, Kumar, Maurya, et al. Quercetin Regresses Dalton's Lymphoma Growth via Suppression of PI3K/AKT Signaling Leading to Upregulation of p53 and Decrease in Energy Metabolism, Nutr. Cancer, 67 (2) (2015) 354-363.

[22] M. Granato, C. Rizzello, M. S. Montani, Quercetin induces apoptosis and autophagy in primary effusion lymphoma cells by inhibiting PI3K/AKT/mTOR and STAT3 signaling pathways, J. Nutr. Biochem, 41 (2017) 124-136.
[23] C. F. Spink, L. J. Keen, F. K. Mensah, et al. Association between non-Hodgkin lymphoma and haplotypes in the TNF region, Br. J. Haematol, 133 (3) (2006) 293-300.

[24] B. Nedoszytko, B. Olszewska, J. Roszkiewicz, et al. The role of polymorphism of interleukin-2, $-10,-13$ and TNF- $\alpha$ genes in cutaneous T-cell lymphoma pathogenesis, 33 (6) (2016) 429-434.

[25] Y. K. Kim, J. S. Shin, M. H. Nahm, NOD-Like Receptors in Infection, Immunity, and Diseases, Yonsei. Med. J, 57 (1) (2016) 5-14.

[26] L. C. Chiang, W. Chiang, M. Y. Chang, et al. Antileukemic activity of selected natural products in Taiwan, Am. J. Chin. Med, 31 (01) (2003) 37-46.

[27] S. H. Park, S. Ham, T. H. Kwon, et al. Luteolin induces cell cycle arrest and apoptosis through extrinsic and intrinsic signaling pathways in MCF-7 breast cancer cells, J. Environ. Pathol. Toxicol. Oncol, 33 (3) (2014) 219-231.

[28] R. Gharbaran, E. Shang, O. Onwumere, et al. Luteolin Induces Cytotoxicity in Mix Cellularity Classical Hodgkin's Lymphoma via Caspase Activated-cell Death, Anticancer. Res, 40 (9) (2020) 4907-4912.

[29] M. Imran, A. Rauf, ZA. Shah, et al. Chemo-preventive and therapeutic effect of the dietary flavonoid kaempferol: A comprehensive review, Phytother. Res, 33 (2) (2019) 263-275.

[30] C. Liu, F. Gao, B. Li, et al. TLR4 knockout protects mice from radiation-induced thymic lymphoma by downregulation of IL6 and miR-21, Leukemia, 25 (9) (2011) 1516-1519.

[31] MK. Kim, C. Suh, HS. Chi, et al. VEGFA and VEGFR2 genetic polymorphisms and survival in patients with diffuse large B cell lymphoma, Cancer. Sci, 103 (3) (2012) 497-503.

[32] P. Salven, A. Orpana, L. Teerenhovi, et al. Simultaneous elevation in the serum concentrations of the angiogenic growth factors VEGF and bFGF is an independent predictor of poor prognosis in non-Hodgkin lymphoma: a single-institution study of 200 patients, Blood, 96 (12) (2000) 3712-3718.

[33] M. Blonska, Y. Zhu, H. H. Chuang, et al. Jun-regulated genes promote interaction of diffuse large B-cell lymphoma with the microenvironment, Blood, 125 (6) (2015) 981-991.

[34] F. Bouchard, SD. Bélanger, K. Biron-Pain, et al. EGR-1 activation by EGF inhibits MMP-9 expression and lymphoma growth, Blood, 116 (5) (2010) 759-766. 